\title{
DESARROLLO COGNITIVO E INTERACCIÓN ASIMÉTRICA: NOTAS PARA SU ESTUDIO MICROGENÉTICO
}

\author{
Sylvia Sastre i Riba \\ Estanislau Pastor i Mallol \\ Universitat "Rovira i Virgili" de Tarragona
}

\begin{abstract}
RESUMEN. Estudiar el desarrollo cognitivo individual supone tener en cuenta las interacciones sociales que, parcial o totalmente, lo condicionan y controlan.

Desde un perspectiva neoconstructivista tripolar, se reflexiona sobre los fundamentos teórico-metodológicos que deberían guiar su investigación. Surgen cuestiones como: ¿donde está el sujeto en la Psicología del Desarrollo Cognitivo actual? ¿cuál es su papel en la interacción?, ¿cómo incide su actividad en la organización de la interacción, y ésta en la actividad del primero?, ¿qué condiciones o tipos de interacción se establecen y cuáles son sus efectos en el progreso cognitivo individual?.

Una de las investigaciones realizadas ejemplifica estas reflexiones, mostrando mediante la aplicación del método microgenético sus ganancias y limitaciones, destacando entre ellas la necesidad de: -optimizar los análisis, -precisar los intercambios indirectos y los mecanismos de control que los niños pueden utilizar en ellos; - precisar los grados de participación e intercambio de los partenaires como función de la dinámica que se establece.
\end{abstract}

ABSTRACT. Interaction is essential to study Cognitive Development. From a tripolar neoconstructivist point of view, we urge the necessity to discuss the meaning of "interaction" and the methodological bases studying it.

Some questions arise: where is the infant in Cognitive Developmental Psychology today?, what is the infant's influence on interaction and viceversa? what is the effect of different kinds of interaction on cognitive development?, what interactional types and conditions could be established and what are their effects they have on individual cognitive development?.

Empirical data from one research carried out are presented as a brief example of research into and reflections about these questions. Using microgenetic method, the results show us the possibilities and limitations of studyng and understanding the influence of interaction on cognitive development.

\section{Introducción}

Actualmente, estudiar el desarrollo cognitivo del sujeto supone, tener en cuenta las interacciones sociales que condicionan y controlan, en totalidad o en parte, dicho desarrollo y su funcionamiento (Verba, 1993a). 
Bajo esta premisa, existen actualmente numerosas publicaciones que defienden e intentan demostrar la relevancia de la interacción en el estudio y explicación de la cognición desde su génesis.

Resulta sorprendente constatar como el énfasis puesto en la interacción conduce a afirmaciones reduccionistas e incluso a proponer que desarrollo y aprendizaje podrían ser el mismo fenómeno (Perret-Clermont, 1993).

Sorprende aún más que desde corrientes cercanas a Piaget, por ej. la Psicología Social Cognitiva, se constate un acercamiento más reduccionista al intentar demostrar que la cognición es un fenómeno social, e incluso que las estrategias lógicas de razonamiento son producto de las "rutinas sociales" (Gilly y Roux, 1993). En cambio, desde la corriente que típicamente ha defendido el papel de la interacción en el desarrollo y funcionamiento cognitivo, influida directamente por la obra de Vygotsky, se percibe un progresivo reconocimiento del papel de lo individual (Elbers, 1992).

Todo ello plantea al estudioso de la cognición humana, la necesidad de profundizar en la investigación de las relaciones individual/social, con el fin de conocer el papel de cada uno en un fenómeno único como es el funcionamiento intelectual, más allá de tendencias e interpretaciones reduccionistas en uno $\mathrm{u}$ otro sentido.

Ante el estado actual descrito, desde una perspectiva neo-constructivista tripolar (sujeto-otro-objeto), aparecen cuestiones como: ¿dónde está el sujeto en la Psicología del Desarrollo Cognitivo actual?, ¿cuál es su papel en la interacción?, ¿cómo incide su actividad en la organización de la interacción, y ésta en la actividad del primero?, ¿qué condiciones o tipos de interacción se establecen y cuáles son sus efectos en el progreso cognitivo del sujeto?. Preguntas a las que pretendemos dar respuesta en base a nuestros resultados.

\section{Perspectiva teórica general en el estudio del desarrollo cognitivo y la interacción}

El estudio de la cognición humana tiene ya una larga tradición en Psicología, si bien el estado actual de conocimientos no refleja el esfuerzo invertido para su entendimiento. Existen diferentes interpretaciones sobre lo que denominamos "inteligencia" o "cognición" y su desarrollo, de tal manera que el concepto actual es heterogéneo (Sternberg, 1993).

Las teorias más relevantes (Piaget, Vygotsky, Procesamiento de la Información) nos han ofrecido conceptos explicativos no siempre concordantes y bipolaridades interpretativas, de entre las que cabría destacar el acento en el rol que el individuo o lo social juegan en la inteligencia y su desarrollo. Y no sólo esto sino que dichas posturas han sido típicamente interpretadas como "opuestas" en las publicaciones actuales (Tudge y Winterhoff, 1993).

Por ejemplo, es común la creencia de que, para Piaget, el niño era como un científico que trabajaba él solo, con la ayuda de su capacidad lógica, sobre el material: físico, lógico y matemático del mundo para construir el sentido de la realidad. Es común la creencia de que para Vygotsky la inteligencia y el desarrollo cognitivo eran un proceso social desde el nacimiento, asistido por los otros (adultos o iguales) más competentes en las habilidades e instrumentos provinientes de la cultura. 
No discutiremos aquí la dimensión real de dichas creencias, pero interesa señalar que han dado lugar, desde cada una de las corrientes cercanas a ellos, a diferentes estudios sobre el papel de la interacción en el desarrollo cognitivo, lo cual demuestra su relevancia en la investigación sobre la cognición y su desarrollo.

Desde la vertiente contextualista, estos estudios han tenido distintas fases (Arnay et alt., 1994) que han ido desde la consideración inicial del niño como receptor pasivo (Wood, Bruner y Ross, 1976) con la participación del concepto de andamiaje (o ayuda del adulto en la mejora de las habilidades del niño), hasta un segundo momento en el que se consideró que el andamiaje mejoraba si tenía en cuenta la actuación y tipo de demandas del niño (Elbers, 1991) estudiándose a éste como modulador activo de las situaciones y no como producto de la actividad del adulto. Finalmente, hoy en día, continúa enfatizándose el papel del niño (Elbers, 1992) y se defiende que el desarrollo puede ser el fruto de diversas influencias madurativas, interactivas y sociales.

A nuestro entender es preciso distinguir aún el papel de lo individual y lo social en el desarrollo cognitivo, además, es necesario que los contextualistas actuales establecezcan la distinción entre los individuos y los contextos en los que se sitúan.

Por otra parte, desde la perspectiva piagetiana, se ha introducido el estudio de la interacción desde (Fraysse, 1993):

- La psicología social cognitiva que, ampliando el marco piagetiano, estudia díadas simétricas en conflicto sociocognitivo. Los psicólogos sociales se lanzaron al estudio del desarrollo social de la Inteligencia (Perret-Clermont, 1979; Doise y Mugny, 1981; Mugny, 1985) con el reto de introducir en una perspectiva constructivista la dimensión social. Hoy en día han logrado definir un objeto propio -la construcción social de las representaciones cognitivas- y fundar sus propios instrumentos metodológicos.

- El estudio de los mecanismos psicosociales de los funcionamientos y construcciones cognitivas (Gilly et alt.,1988). Estudian díadas simétricas y las regularidades sociales estructurantes determinadas por las significaciones de la tarea. Se interesan por la cooperación, la co-construcción por conflicto socio-cognitivo, la imitación y la guía por explicitación.

La evolución de estas corrientes (Perret-Clermont, 1993; Gilly y Roux, 1993), ha supuesto un acercamiento contextualista y una pérdida de la individualidad de la construcción individual, llegando a afirmar que desarrollo y aprendizaje son un mismo fenómeno dado el contexto social en el que ambos se sitúan (Perret-Clermont, 1993) y que tareas de tipo lógico, como por ejemplo la distribución, son fruto de la rutina social (Gilly y Roux, 1993).

- La perspectiva pluridimensional (Beaudichon, Verba y Winnykamen,1988) estudia díadas simétricas y asimétricas y las organizaciones asociativas por imitación, tutela y co-construcción. El modelo pone su acento en:

a. Observar al niño en situaciones abiertas (ecológicas)

b. Una aproximación microgenética multidimensional que tiene en cuenta las dimensiones:.temporal: elección de episodios y organización de la actividad en secuencias significativas cognitiva: operaciones de pensamiento sucesivas 
intra-inter sujeto/s. social: función de las conductas sociales en la actividad compartida.

c. Evidenciar las organizaciones interactivas o los niveles de acción integrados.

Esta es, a nuestro entender, la perspectiva que permite en mayor medida un acercamiento al conocimiento del papel de lo individual y lo social en el desarrollo cognitivo en tanto los contempla a ambos en las microgénesis explicativas de los distintos procesos y conocimientos, su evolución y productos.

Por otra parte, según Carugati y Gilly (1993), las nociones utilizadas en el estudio de la interacción han ido modificándose a lo largo del tiempo, desde las nociones de "cooperación-competición", "imitación-conflicto", hacia las referentes a los niveles de articulación "intraindividual-interindividual" que permiten estudiar las regulaciones sociales de los participantes, las transformaciones de sus esquemas de acción, y en general las nuevas coordinaciones cognitivas.

En realidad, las posturas no están lejanas, como tampoco lo estaban las de Piaget y Vygotsky. Más bien son complementarias con una clara tendencia de acercamiento, tanto metodológico como teórico una vez hayan abordado problemas básicos como el de qué es lo que consideran central en el desarrollo cognitivo: las influencias sociales o la estructura interna, o tal vez ambas.

Quedan aún interrogantes esenciales por resolver. Las investigaciones en curso se dirigen describir la interacción e interpretan las conductas del niño en base a su responsividad en una situación social que le envuelve (lo cual conduce fácilmente a decir que su razonamiento o su cognición es social). En cambio, no explican: qué hay de individual y qué hay de social, hasta qué punto el pensamiento reflexivo opera y obtiene unos productos que, sin duda, serán ratificados o no socialmente, pero que para obtenerlos el niño realiza una serie de operaciones no sugeridas o determinadas por el exterior, necesarias para responder o solucionar la tarea.

Si insistimos, como lo hacen la mayoría de estudios actuales, en el rol del otro y los metasistemas de control en las adquisiciones, corremos el riesgo de minimizar el papel del niño en la gestión cognitiva y social.

Y ello no supone postular otra vez la individualidad piagetiana, sino defender que el funcionamiento individual existe, y que sin él difícilmente puede establecerse una interacción productiva, con un producto final distinto al existente en los inicios de la situación, tanto por parte del sujeto como por parte del partenaire o contexto.

Por lo tanto, si no todo es individual ni todo es social, es hora ya de precisar y ahondar en el concepto de interacción y desarrollo cognitivo que utilizamos en nuestros estudios, no sea que lo hayamos perdido y estemos únicamente abordando la externalidad de un proceso complejo en el que el individuo tiene mucho que aportar.

La dificultad, como apunta Verba (1994), reside en la articulación de los niveles intra-individual, interpersonal e institucional en los procesos de conocimiento y en la puesta en evidencia de los mecanismos que dan cuenta del funcionamiento y cambios de la competencia del niño en cada uno de estos niveles.

El problema fundamental es conocer el desarrollo cognitivo, entendido como proceso de actualización y construcción sucesiva de lo que denominamos "inteligencia".

Inteligencia entendida como la capacidad para establecer relaciones que presupone la posibilidad de categorizar y/o estructurar el saber (Lécuyer, 1989). 
En este sentido, existen distintos objetos sobre los que se establecen dichas relaciones (sucesos, acciones, estados, conceptos, personas...), existen diferentes tipos de relaciones (pertinencia, causalidad, analogía, ... ), diferentes tipos de organización de dichas relaciones (niveles de complejidad en la organización de los conocimientos), y existen diferentes instrumentos de aprehensión de dichas relaciones (percepción, lenguaje, ...) que determinan las diversas formas de aplicación de la Inteligencia. Todo ello supone una interacción y adaptación continuada, en feed-back, al medio físico y social en el que el sujeto se encuentra inmerso. Ello significa que la actividad cognitiva es el producto de un sistema de interacciones entre tres polos: sujeto-otro-objeto.

El desarrollo cognitivo sería un proceso que se construye a partir de unas bases biológicas (estructuras preformadas) y lógicas (estructuración y organización de los instrumentos cognitivos y de la información obtenida), en un contexto bidireccional, a la vez individual y social.

En dicho proceso es indispensable conocer, desde el principio de la vida: tanto el tipo de mecanismos, operaciones, funciones, instrumentos, etc. que el sujeto pone en juego (para captar, elaborar, estructurar, conferir significación a la información y generar una respuesta), como el producto obtenido.

Si esto es así, es preciso plantearse la naturaleza de la inteligencia y los pre-requisitos o las disponibilidades intelectuales existentes en el organismo humano desde el nacimiento. Es decir, las relaciones entre Biología e Inteligencia.

Sin esgrimir tesis innatistas, las relaciones entre Biología e Inteligencia van concretándose cada vez más (Carey \& Gelman, 1991), generando un renovado interés. Actualmente se ha redescubierto el papel de la Biología en el desarrollo cognitivo que podría sintetizarse en tres formas: 1.como especificación del estado inicial, 2.como la consideración de la influencia que tienen los factores madurativos en el desarrollo y, 3.como consideración de la relación existente entre evolución y cognición. Sea como sea, no podemos estudiar la inteligencia y el desarrollo cognitivo sin tener en cuenta sus bases biológicas.

Estamos de acuerdo en que tanto la estructura biológica como la estructura intelectual, son contribuidoras fundamentales en la epigénesis de la mente. Esto, lejos de acercarse al innatismo tradicional da un nuevo sentido al desarrollo cognitivo en tanto se parte de la existencia de unas estructuras preformadas (necesariamente biológicas) (Mounoud, 1992) que sirven como base o plataforma para el despliegue intelectual al proporcionar al bebé los instrumentos necesarios para empezar a captar y elaborar, -organizada y significativamente-, la información y los primeros conocimientos .

En esta línea, es esencial investigar los primeros momentos del desarrollo cognitivo. Concretamente, los tres primeros años de vida son fundamentales para dar cuenta de la génesis de los mecanismos y procesos intelectuales necesarios para el funcionamiento cognitivo posterior, destacando la precocidad de la competencia del bebé (Mandler, 1992, Spelke, 1991, 1994).

Si el problema fundamental reside en conocer el desarrollo cognitivo del niño entre 0;0-3;0 años, el abordarlo requiere tener en cuenta dos cuestiones base -que trataremos específicamente en el siguiente apartado-:

1. la fundamentación teórica de los conceptos intervinientes;

2. la decisión metodológica según el tipo de fenómeno que pretendemos conocer. 


\section{Una propuesta teórico-metodológica en el estudio del desarrollo cognitivo e interacción}

$\mathrm{Si}$, de acuerdo con lo expuesto, el estudio del desarrollo cognitivo en contextos interactivos requiere atender a la fundamentación teórica del fenómeno a estudiar y a la decisión metodológica, debemos analizar cada uno de estos puntos.

\subsection{Requisitos teóricos}

Hay que delimitar qué debemos apresar para explicar el fenómeno cognitivo y la incidencia que en él tiene la interacción. Para ello, proponemos atender a una doble vertiente:

A. Apresar el funcionamiento lógico, entendiéndolo como un organizador del pensamiento, a la vez que un conjunto de instrumentos que permiten al sujeto operar sobre el mundo (Langer, 1990, Houdé, 1994).

Esto supone una relación directa con la acción organizada dentro de lo que Piaget denominó "Lógica de Significaciones" (Piaget y García, 1987) efectiva antes de que el grupo INRC entre en funcionamiento y más allá de conceptos ortodoxos y poco explicativos como el de equilibración (no referido tanto a la organización y funcionamiento lógico del pensamiento, como al proceso de desarrollo cognitivo general). Se trata, pues, de la lógica en acción de los niños durante los primeros años de vida que según dicho autor contiene las dieciseis operaciones binarias características de la lógica formal. Otros autores como por ejemplo Langer $(1986,1990)$ la han denominado "protológica" mostrando a través de su funcionamiento la arquitectura mental del bebé.

La primera cuestión a resolver es la de: ¿Por qué en un estudio del desarrollo cognitivo nos centramos en la Lógica?, ¿acaso no existen otros sistemas intelectuales para caracterizar dicho desarrollo?.

Hay diferentes postulaciones de investigadores actuales sobre el papel de la respresentación en el desarrollo cognitivo (Karmiloff-Smith, 1992; Mandler, 1992; Mounoud, 1992, etc.), en cambio se ha prestado poca atención a los orígenes y el papel de la lógica en la infancia.

Desde nuestro punto de vista, la acción' (física o mental) es el instrumento base que utiliza el sujeto para construir su inteligencia. Desde el principio el niño "actúa" sobre su entorno, obteniendo así la información necesaria para ir construyendo su conocimiento sobre el mundo y elaborando nuevas formas de pensamiento.

Si esto es así, "actuar" no es suficiente sino que hay que actuar con sentido, organizadamente, y ahí interviene la lógica en tanto organiza y da sentido a la actividad y a los conocimientos que de ella se derivan (Langer, 1990).

Como Langer (1990) señala, la estructura lógica caracteriza toda la cognición. Si, de acuerdo con la definición que hemos propuesto, la inteligencia supone desde su inicio el establecimiento de relaciones, requiere de una organización tanto de dichas

1. Acción entendida como actividad organizada 
relaciones (tipo, nivel de complejidad..), como de los recursos que utiliza. Por lo tanto, de la lógica.

Esta aproximación, decididamente constructivista, defiende la racionalidad del bebé y demuestra la existencia de componentes fundamentales de la vida psicológica que aseguran desde los primeros meses un entorno coherente e inteligible. Este entorno se elabora mediante la aplicación pragmática (en el curso de la acción) de la cognición lógico-matemática en la cual se distinguen tres estructuras-base:

1. la estructura combinatoria que comprende la realización pragmática de las operaciones de: composición, descomposición y deformación;

2. la estructura relacional que comprende cuatro operaciones pragmáticas: adición, sustracción, multiplicación y división;

3. la estructura condicional que comprende tres tipos de operaciones pragmáticas: intercambio, correlación y negación.

El producto de dichas estructuras pragmáticas constituye los elementos fundacionales de la cognición lógico-matemática y consiste en la elaboración de: objetos, colecciones y series, estableciendo en ellas relaciones de cuantificación, equivalencia y no-equivalecia (consultar para más detalle: Langer, 1980, 1986, 1990, 1995; Houdé, 1994; Pastor y Sastre, 1994).

De esta manera, la cognición sería el producto de la acción sobre el entorno que ofrece sus propiedades físicas y sociales sobre las que el sujeto actúa. Igualmente importante es la observación que el sujeto hace de sus acciones y resultados; así pues, la inteligencia sería, en buena medida, el producto de la relación entre las propias acciones y los resultados obtenidos sobre el entorno físico y social. Por lo tanto, las primeras construcciones intelectuales progresan a partir de la intersección de dos formas de interacción: la primera es su interacción con el entorno físico y social, la segunda es la interacción con las construcciones cognitivas previas y su organización.

De ahí que, a pesar de reconocer que el sujeto humano es un ente "simbólico" (Rivière, 1990), con una capacidad de interiorización y categorización que le permite desprenderse de la dependencia física directa de la realidad, yendo "más allá de la información dada" (Bruner, 1988), creemos que es también una entidad eminentemente "lógica", es decir, que desde su nacimiento percibe, confiere significación y estructura relacional a los objetos, eventos y personas del entorno, operando sobre ellos.

A lo largo del desarrollo cognitivo, ambos sistemas intelectuales-base funcionarán (en su sentido óptimo) interconectadamente, de forma que será difícil de concebir uno sin el otro o distinguirlos netamente en una operación intelectual. Pero tal vez, sea la capacidad lógica la impulsora de la necesidad representativa (Langer 1980, 1986), ya que si el sujeto es incapaz de establecer relaciones coherentes, organizadas y significativas entre las informaciones que recibe de forma perceptivo-motora, difícilmente podrá "interiorizar" datos-base para la progresiva construcción intelectual.

Por lo tanto, estudiar el desarrollo cognitivo durante los tres primeros años de vida, reclama conocer la estructura lógica pragmática (en acción) que el niño aplica y construye, qué tipo de operaciones y funciones inherentes a su actividad intencional precoz realiza (Langer,1990), cómo elabora los significados y el conocimiento y cómo estructura su acción en secuencias cada vez más complejas. 
En este proceso, la interacción que el niño establece con el medio, es de tipo físico (dominio de la acción hacia la organización y el conocimiento lógico) y social. De la interacción física, hemos discutido y fundamentado el papel de la lógica en su estructura y organización. La interacción social es tratada en el siguiente apartado.

B. Apresar los requisitos, tipos y condicionantes de la interacción .

Es preciso conocer las condiciones en las que se produce la interacción social, los tipos y requisitos que toma con el fin de asegurar que, realmente, el producto resultante sea algo nuevo a lo que el sujeto podría producir solo y, por lo tanto, lleve a una ganancia.

Ello supone reflexionar y profundizar funcionalmente en su significado, más allá de aproximaciones superficiales. Supone, básicamente, entender el rol y aportaciones de cada uno de los participantes en la tríada: sujeto-objeto-otro.

El concepto de interacción que fundamenta nuestras investigaciones, desde una perspectiva constructivista pluridimensional, considera que el niño es poseedor de una disponibilidad y potencialidad propias, y que construye su inteligencia en base al intercambio continuado con el medio, en una relación de "feed-back" y de regulación mutua con otros adultos o iguales.

Esta construcción se realiza en doble dirección: desde el sujeto hacia el medio y del medio hacia el sujeto, destacando la capacidad de autoregulación del individuo respecto a aquél. Por lo tanto, el desarrollo cognitivo se realizaría a través de lo individual pero en relación con situaciones de intercambio social, básicamente las de: imitación, tutela y cooperación (Verba, 1994).

Eludimos con ello las connotaciones behavioristas de pasividad del organismo frente al medio, o del determinismo soviético.

Introducimos la precisión de que el desarrollo cognitivo consiste en "una estructuración progresiva de las relaciones con el ambiente, estructuración que también es individual" (Mugny y Pérez, 1988), lo cual significa que la interacción social no sustituye a las actividades individuales del sujeto, pero contribuye a su organización. Desde este punto de vista, las nociones de "intersubjetividad" (Rogoff, 1990; Werstch, 1985) y significación compartida no son fruto de un proceso interactivo en el que el elemento interpsicológico se convierte en intrapsicológico, sino que tanto la una como la otra parten y dependen de las capacidades individuales de cada sujeto (intrapsicológico).

Desde esta Psicología tripolar (sujeto-otro-objeto), el individuo continúa siendo esencialmente activo en su desarrollo, integrando el medio social como moldeador de sus capacidades (sin determinismo).

Si, como nos muestran los resultados de investigaciones anteriores (Sastre, 1991; Sastre y Pastor, 1994), el sujeto a partir de su actividad es capaz de incidir en el tipo y forma de interacción, contribuyendo al éxito o facilidad de ajuste del otro, es preciso estudiar funcional e interactivamente quién participa y de qué forma, y cómo se va desarrollando el proceso de intercambio y condicionamiento mutuos.

Esto requiere romper determinismos e integrar a todos los participantes. Requiere también considerar un aspecto altamente olvidado en la investigación actual que es el rol del objeto y sus propiedades en todo este proceso. Es decir, afrontar la teoría del objeto y concretamente de la significación del objeto. Significación no solo social, sino de 
las propiedades físicas y lógico-matemáticas que debe descubrir el niño y que conforman, junto con lo social, la significación total. Por lo tanto, significación como base del conocimiento, y éste no sólo es social sino también físico y lógico-matemático.

\subsection{Fundamentos para la decisión metodológica}

Profundizar en los postulados anteriores, requiere una decisión metodológica que se ajuste a las características del fenómeno a estudiar (Sastre y Pastor, 1995, Pastor y Sastre, 1995) y que permita conocer el rol de cada uno de los participantes en el proceso (sujeto-objeto-otro), dentro del continuum temporal en el que se produce.

Pero... ¿cómo acceder al desarrollo cognitivo individual?, ¿cómo conocer en qué medida y de qué forma dicho desarrollo influencia y se ve influenciado en un contexto de interacción?.

No podemos olvidar que nos situamos frente a procesos que no son siempre inmediatamente perceptibles y evidentes y que el tiempo es un aspecto central en la organización del comportamiento. Las interacciones están estrechamente ligadas a problemas generales de análisis de la organización temporal, tanto del comportamiento verbal como no verbal, intra e interindividual.

La decisión metodológica comporta un serio problema ya que en numerosas ocasiones conocemos lo que queremos estudiar pero no sabemos cómo hacerlo, improvisando o aplicando tentativamente metodologías de análisis que no siempre se adecuan a los fenómenos que debemos apresar. Por ello, recogiendo las ideas hasta ahora expuestas, es importante preguntarse por el "qué" y el "cómo".

¿Qué se ha de apresar en el estudio?:

a. la acción del niño y su organización lógica. Es preciso "meterse en el corazón" de las microgénesis y ver qué problemas se plantea el niño y cómo los resuelve, a partir de los observables, centrándose sobre las secuencias de acciones y su encadenamiento, elucidando las relaciones entre sistemas de comprensión y procedimientos de descubrimiento (Inhelder y Caprona, 1992);

b. la interacción, y dentro de ella:-los pre-requisitos cognitivos, -la naturaleza de los intercambios, -la motivación, y -las variables de significación.

¿Cómo podemos apresarlo?. El método que posibilita llevar a cabo dicho análisis es el método microgenético, funcional. Este método permite, a partir de la actividad inicial del sujeto, el seguimiento secuencial de su organización (análisis cualitativo, secuencial de la actividad y su contenido: origen y desarrollo de las estrategias lógicas resolutivas y proceso de significación), así como de los patrones de interacción asimétrica que se van estableciendo y modificando en el continuum temporal (roles, tipos, condiciones y efectos de la regulación, ajuste e intersubjetividad); finalmente, a partir de la actividad inicial del sujeto y la interacción establecida, podemos conocer cómo dicha actividad va modificándose (Verba, 1993b; Sastre y Pastor, en prensa).

El porqué de la elección de una metodología cualitativa de análisis se justifica a partir de los puntos que sugiere Saada Robert (1992) y que compartimos plenamente: 1.-el funcionamiento del saber descansa sobre procesos cualitativos que sólo un análisis profundo y detallado permite reconstituir, 2.-el desarrollo de la resolución (pro- 
yecto, medios, solución) forma una totalidad indivisible dado que se busca su coherencia interna; 3.- el desarrollo de la solución supone un análisis temporal de los datos basado sobre índices observables a la vez sincrónicos (conjunto de índices pertenecientes a un momento $X$ ) y diacrónicos (un mismo índice, aparecido en distintos momentos de la resolución). (Para una discusión más amplia sobre el tema, ver: Sastre y Pastor, en prensa).

Para diseñar coherentemente la metodología descrita para un objetivo concreto de investigación es preciso atender especialmente:

a. al tipo de tareas que proponemos al sujeto, las cuales: .facilitarán un interés prolongado en la resolución de la tarea, posibilitarán el ejercicio de las actividades cognitivas implicadas, presentarán un nivel de dificultad real pero asimilable por el niño y, adquirirán el sentido de "problema";

b. el tipo de material, sus propiedades y posibilidades, no todo material permite al niño ejercitar todo tipo de acción (material abierto/cerrado, figurativo/no-figurativo...);

c. el rol del "otro", atento al niño, dejándole la iniciativa o siguiendo sus requerimientos;

d. -el registro de la actividad, preferiblemente en vídeo ya que nos permite retornar tantas veces como sea necesario a puntos cruciales de la actividad, revelando los ritmos de las conductas, sus modificaciones, e inferir los modelos subyacentes y su organización funcional;

e. el análisis del contenido debe permitirnos conocer cómo el niño se representa la situación (y le atribuye significación) y qué actividad organiza; debe permitirnos conocer también de qué parte el "otro", es decir: qué tipo de representación (o teoría) de la tarea, la situación y el niño le guía en su intervención, y cómo el objeto condiciona dicha actividad e interacción.

La aplicación del método microgenético, cualitativo, puede complementarse con el uso de programas de análisis elaborados específicamente para tratar datos cualitativos, por ejemplo, "Thème" (Magnusson, 1993).

Este programa ha sido desarrollado para detectar patrones de conducta interactiva desde una perspectiva ecológica y estructural, utilizando la lógica de tiempo real. Permite integrar el estudio de la organización secuencial y el de la organización sincrónica entre dichos patrones (para más información, ver: Magnusson, 1993, Pastor y Sastre, 1995).

Contemplados hasta aquí los fundamentos que sirven de guía a una decisión teórica y metodológica sobre el desarrollo cognitivo y la interacción, exponemos a continuación el resumen de una investigación reciente que ejemplifica los razonamientos antes aportados. Nuestro objetivo es el de poner en práctica todas estas aportaciones en el marco de una investigación concreta.

UN Estudio EMPÍRICO del proceso de significación y organización del "scaffol$\operatorname{ding}^{\prime \prime}(0 ; 11-0 ; 18 \text { a.) })^{\prime \prime}$ (Sastre y Pastor, 1994)

Se abordó aquí el problema del desarrollo cognitivo y dentro de él, la lógica de significaciones (Piaget y García, 1987), integrando la tripolaridad mencionada: sujeto objeto(significación) -otro ("scaffolding") en torno a: 
1. El concepto y el papel de la significación en el desarrollo cognitivo y en la construcción del conocimiento.

2. El papel del "scaffolding" en su construcción, dada su relevancia en las situaciones de interacción al posibilitar, en mayor o menor grado, el proceso de interiorización.

Por lo tanto, al marco teórico de referencia que hemos expuesto en el apartado anterior (ver apartado 1 "requisitos teóricos"), deberán añadirse los conceptos específicos de este estudio que, de manera breve exponemos seguidamente:

1. Significación: Es el resultado de la asimilación del objeto considerado a un esquema del sujeto y recíprocamente, siendo toda asimilación fuente de significación (Piaget y García, 1987).

Implica la actividad del niño en interacción física o mental y supone siempre una abstracción reflexiva ya que las propiedades del objeto no son observables puros sino que constituyen una interpretación de los datos.

Por ejemplo, si un objeto " $x$ " está ubicado sobre un soporte " $y$ " que permite atraerlo hacia sí, o bien está ubicado al lado o lejos de ese soporte, habrá implicación entre significaciones si el sujeto "comprende" que en este segundo caso de nada sirve atraer " $y$ "; la relación o acción "ubicar sobre" adquiere por este hecho la significación de una razón necesaria.

La significación existe primero a nivel de predicados o conjunto de similitudes y diferencias entre una propiedad observable y las otras registradas simultáneamente o ya conocidas. Más tarde, la significación existe en los enunciados.

El ámbito de la significación se concreta en:

a. Significación del Objeto: es el conjunto de predicados equivalentes a "lo que se puede hacer con él" (asimilación a un esquema de acción).

b. Significación de la Acción: es "lo que se llega a hacer con ella", en función de la transformación que produce en los objetos o situaciones.

La significación es esencial para el control de la actividad del niño y para la comprensión de los mecanismos intervinientes en la formación microgenética del conocimiento en ciertos contextos.

Es un elemento básico dado que sin ella no hay actividad con objetos, no hay información, ni organización, ni generación de nuevos esquemas, ni tampoco deducciones lógico-matemáticas, ni conocimiento físico. Si el niño no comprende la tarea o no elabora una significación sobre ella, los objetos intervinientes y la situación, difícilmente podrá estructurar un curso de acción a través de proyectos de acción que se modifiquen a medida que vaya generando nuevas significaciones $y$, por lo tanto, nuevas posibilidades o necesarios de la actividad.

Su función, como hemos precisado, es la de guiar y determinar la actividad infantil y las operaciones o funciones a realizar (Inhelder y Caprona, 1992).

La significación está relacionada con la Teoría del Objeto (Lehalle, 1990), la resolución de problemas (Gréco, 1991) y con las estructuras lógicas. Esta concretización no supone en ningún momento despreciar la significación social del objeto, no estudiada en esta investigación. Entendemos que la significación propuesta refleja -o puede reflejar- en mayor medida la actividad y elaboración intelectual del sujeto en tanto está estrechamente vinculada a sus posibilidades. 
2.-"Scaffolding": Si la inteligencia es entendida como una construcción individualsocial, postulamos: 1) la doble dirección del desarrollo cognitivo "inside-outside" y "outside-inside"; 2) la necesidad de: intersubjetividad (Werstch, 1985), regulación mutua y de un "feed-back" entre: acción del niño - información del adulto - acción del niño como respuesta al adulto (Sastre y Pastor, 1992). El "scaffolding", por lo tanto, no substituye a la acción individual pero puede contribuir a su organización y proyección.

Hipotetizamos que el "scaffolding" puede verse favorecido y condicionado no sólo por estos factores sino también por el proceso de atribución de significado al material (propiedades y posibilidades) y especialmente por el modo cómo el niño organiza su acción con las repercusiones que ello supone en la percepción-interpretación del otro.

Esto significaría que el "scaffolding" se mantendría por la organización de la acción del niño a través de los proyectos de acción, modularizados paso a paso, de acuerdo con los resultados obtenidos.

Para poder estudiar el problema que planteamos, seguimos la descripción metodológica que resumimos en los Cuadros $n^{\circ} 1$ y 2 .

Método:

- Población: $n=9$ niños normales de edades comprendidas entre $0 ; 11-1 ; 6$ años. Nivel socio-económico medio.

- Selección de los sujetos: voluntarios, tras haber enviado un tríptico explicativo a diversas Guarderías. Se evaluó su nivel de desarrollo mediante las Escalas Cambrodí-Sastre (1993).

- Condiciones de la observación sistemática

- Situación: Registro de la actividad espontánea con material abierto, no figurativo. Adulto experto conocido en interacción con el niño.

- Tiempo: 10-15 minutos. Cada mes.

- Lugar: en casa del niño

- Consigna utilizada: interactuar con el niño, a partir de su iniciativa y favoreciendo la generación de proyectos de acción y el descubrimiento de propiedades del material

-- Microgenético:

- Estudio longitudinal. Funcional.

\section{Cuadro $\mathrm{n}^{\circ}$ 1. Metodología}

Lo que pretendíamos apresar era:

- el proceso de significación y los pasos sucesivos que el niño va dando en su construcción;

- cómo el adulto en distintos momentos de interacción incide en la actividad del sujeto adaptando su información a los niveles de significación que el niño está utilizando o elaborando;

- el resultado de dicho proceso: cómo se ajusta el adulto y qué repercusiones tiene en el desarrollo de la actividad infantil.

Para conseguirlo, era indispensable poder detectar "patterns" o patrones de conducta individual e interactiva. De ahí que fuera necesario elaborar unas categorías referidas a: 
- la acción del niño

- los niveles de significación infantil

- la información que el adulto ofrece durante la interacción,

- la significación mediada que el adulto utiliza en su información,

- el resultado de todo este proceso en la acción infantil, por tanto, la existencia de ganancias o no en cuanto a la actividad y la significación.

Otorgamos gran importancia al laborioso proceso de categorización (categorías excluyentes), mediante acuerdo entre jueces, ya que determinaría en gran medida el tipo de resultados a obtener.

El proceso de categorización realizado responde a un detallado análisis de la realidad de la cual se parte y a la cual nos remitimos constantemente para elaborar dichas categorías.

Definidas y construidas las categorías (ver Anexo 1 y 2$)^{2}$, el paso siguiente consistía en categorizar, paso a paso, la secuencia de acciones registrada en vídeo que posteriormente sería analizada mediante la aplicación del programa Thème para el análisis de datos cualitativos; por lo tanto, no es una muestra de los tipos de análisis microgenéticos cualitativos que en otros estudios hemos realizado (ver: Sastre y Pastor, 1992; Sastre y Pastor, 1993).

Véase en el Cuadro $\mathrm{n}^{\circ} 2$ el proceso de análisis e interpretación seguido.

\section{Análisis:}

1. Construcción de categorías excluyentes a partir de la reaiidad mediante acuerdo entre jueces

2. Búsqueda de patterns interactivos mediante el programa "Thème" (Magnusson, 1993)

Interpretación :

1. Relación existente entre la actividad del niño y el proceso de significación. Permite analizar el cambio de la acción en sí misma y cómo crece el conocimiento del objeto, revertiendo en un incremento de la actividad (mayores posibilidades de acción) comparativamente de 0;11 y 1;6 a.

2. Relación entre los informacións del adulto y el proceso de significación del niño.

- ganancias en significación y resultados de la acción en relación con la información del adulto.

- no-ganancias en significación y resultados de la acción en relación con la información del adulto.

- desarrollo de "patterns" desde 0;11 a 1;6 meses.

Cuadro $n^{\circ}$ 2.-Analisis de Datos y Proceso de Interpretación de los resultados

Los datos siguieron el tipo de interpretación señalado en el Cuadro $n^{2} 2$. A modo de ejemplo presentamos algunos de ellos ${ }^{3}$, como ejemplo del tipo de resultados obtenidos (recordemos que la finalidad de este artículo no es tanto la de la difusión de resultados como la reflexión en el estudio del fenómeno que nos ocupa).

2 y 3. Tomado del texto: Pastor, E. y Sastre, S. (1995).- Patrones de interacción adulto-niño en la construcción del significado: aplicación del programa "Thème". En: M̃ T. Anguera (dir.) Metodología Observacional. Vol.III. Barcelona: P.P.U. 
En cuanto al punto $n^{0} 1$ de la interpretación, es decir la actividad del sujeto, podemos observar de qué niveles de significación parte el niño y hasta dónde llega, comparativamente con el tipo de significación que el adulto utiliza. Por lo tanto, tenemos ya un indicativo del nivel de elaboración infantil y si se produce o no ajuste entre niño-adulto).

Véase a modo ilustrativo el cuadro resultante de los niveles de significación comparativos ente el niño y el adulto, a los $0 ; 11$ a y los $1 ; 6 a$. .

***Diferencias:

Mínimo nivel de significación:

Máximo nivel de significación:

Tipo de significación:

\section{0;11a.}

niño $\quad \mathrm{snO}^{5}$

adulto sa3

niño sn4

adulto sa6

en acción
$1 ; 6 a$.

sn 4

sa7

sn6

sa7

verbal

\section{Cuadro $n^{2} 3$. Niveles de Acción - Significación}

En cuanto al punto no 2 del proceso de interpretación, analizando comparativamente los datos correspondientes a los sujetos de $0 ; 11$ a. con los de 1;6a., pudimos deducir datos sobre el contenido de la acción del niño (sujeto), el proceso de significación (objeto) y los resultados que se obtienen (de ganancia o no) en la interacción con el adulto (otro)(ver Sastre y Pastor, 1994). Globalmente, estos datos pueden reducirse a unas similitudes y diferencias que precisamos a continuación.

Principales Similitudes $(0 ; 11-1 ; 6$ años):

- la significación guía y organiza la actividad del niño, sus posibilidades y los resultados obtenidos (conocimiento)

- el niño no puede actuar con los objetos si no los "entiende", si no tiene una significación sobre sus propiedades o posibilidades

- la exploración es la primera acción. Tan pronto como el niño encuentra significación (primeros necesarios), cambia hacia la experimentación y la generalización, ampliando los primeros necesarios hacia nuevas posibilidades del objeto $y$, finalmente, nuevos necesarios;

- el ajuste del adulto al proyecto de acción del niño y su importancia para el progreso de la actividad;

- el rol del adulto en términos generales: de confirmación y/o de ofrecimiento de nuevas posibilidades .

Principales diferencias $(0 ; 11-1 ; 6$ años):

1. El nivel de organización de la actividad y su contenido, según los niveles de significación

2. Las "informaciones" del adulto y el proceso de significación del niño .

Cuadro $n^{\circ} 4$. Relación entre la información del adulto y el proceso de significación del niño.

4. Tomado del texto: ver nota anterior. Esta investigación fue presentada en: Sastre, S. y Pastor, E. (1994). Action, Signification process and Scaffolding organization. Poster presented to the XIIIth Biennal Meetings of ISSBD. Amsterdam. ( su publicación está en preparación).

5. Ver Anexo $n^{\circ} 1$. Código de categorías. 
En conclusión, los resultados muestran que el "scaffolding" es favorecido por la organización de la actividad infantil mediante proyectos de acción consistentes y modularizados, paso a paso, de acuerdo con las informaciones obtenidas. Esto demuestra que la estructura cognitiva del niño y la significación de la actividad son necesarias. Pero el "scaffolding" depende también de la comprensión del adulto de las características y posibilidades de los objetos y de las situaciones (significación) así como de la comprensión del proyecto de acción del niño.

Concretamente se demuestra :

- la necesidad de un doble ajuste del adulto: al proyecto de acción infantil y a su nivel de significación, no sólo debe dar información relacionada con lo que el niño realiza (acción), sino que el contenido debe ser similar;

- la necesidad de situarse en la zona proximal de significación para favorecer ganancias en la significación infantil; .

- el rol del lenguage como pivote final en la interiorización de la significación: etiquetar resultados...;

- la relación entre: significación, operaciones lógicas, organización de la acción y conocimiento, no sólo interesa lo que hace el niño (por ej., una composición), sino también que posibles y necesarios extrae (significación) y como inciden en la modularización continuada de la actividad;

- el No conocimiento del objeto y la inexistencia de una significación inicial dificultan el scaffolding y pueden desfavorecer las ganancias del niño (demuestra que el adulto no puede ajustarse a lo que no conoce). Un buen conocimiento y significación del objeto por parte del niño, favorece el scaffolding y las ganancias;

- la importancia y utilidad del método de análisis microgenético para un acercamiento funcional a la conducta individual y social con el fin de conocer su génesis y curso.

Todo ello nos conduce a postular y defender la capacidad autoreguladora de lo individual frente a lo social, es decir, la interacción depende también de la aportación del sujeto que participa en la regulación de ésta y en las posibilidades de interiorizar y elaborar las informaciones transmitidas, sin olvidar las autogeneradas.

\section{Conclusiones relacionadas con los problemas planteados}

En este documento, hemos planteado desde su inicio dos problemas básicos, referentes al sentido y alcance de la interacción en el desarrollo cognitivo, y cómo estudiarlo.

Los resultados de la investigación presentada, enriquecen dichos planteamientos y ofrecen datos para ampliar su interpretación. Globalmente, los resultados obtenidos favorecen:

- la mejor comprensión del proceso de significación y su construcción en el niño, es decir, cuál es el nivel inicial y el proceso de elaboración de nuevos niveles de significación, sus problemas y posibilidades;

- las repercusiones que tiene este proceso en la organización de la actividad y la construcción de conocimiento; 
- el rol de la interacción como mecanismo de cambio cognitivo, en qué momentos y con qué condiciones favorece el cambio y avance significativo y en cuales no, sin substituir el papel del sujeto, es decir, podemos conocer qué rol tiene cada participante;

- el papel de ajuste del adulto a las disponibilidades del sujeto y cómo se adecua a los niveles de significación de los que parte el niño y que le sirven de plataforma para su intervención (en qué punto debe intervenir y cómo);

- qué consecuencias tiene en el resultado de la actividad infantil el tipo de ajuste e intervención del adulto (positiva o negativa);

- el ajuste metodológico a las necesidades del estudio y sus limitaciones;

- el proceso de categorización requiere una exhaustiva reflexión, estructuración y operacionalización del problema en estudio (la significación y la interacción).

En concreto, y en respuesta a las cuestiones previas con las que iniciamos el artículo, argumentos lo siguiente :

1. La primera cuestión hacía referencia a: "¿dónde está el sujeto (niño) y cuál es su papel en la interacción?". Podemos responder a ella afirmando que:

a) si no existe un nivel de significación mínimo de partida por parte del niño los procesos de interacción no son posibles;

b) debe existir, en la situación interactiva, una distancia mínima y máxima entre el nivel de significación del niño y el del adulto (ver cuadro no3). De no ser así, la eficacia de la intervención adulta desciende o se anula. Ello exige que el adulto esté atento al sujeto y a sus niveles de significación (y elaboración).

Aunque se afirme que el proceso de significación es social, debe tenerse en cuenta al interlocutor $y$, en este caso, el adulto debe partir de las características individuales del sujeto quien, finalmente, debe seleccionar, escoger y elaborar la información.. Por estas razones podemos afirmar que, si menospreciamos el papel del propio sujeto en los procesos constructivos, la co-construcción social del saber no sería posible.

El proceso interactivo puede ser iniciado por el niño, a través de su actividad. La exploración es el punto de partida de la acción y el niño la realiza por sí mismo, sin intervención del adulto, mediante la aplicación de esquemas de acción disponibles a través de los cuales busca información que le permita acercarse a la significación del objeto, en un principio desconocido. Es evidente que el papel del niño en este tipo de situación no depende de la interacción sino de él mismo.

El adulto tiene la posibilidad de ofrecer información, canalizarla, ampliarla... pero siempre a partir de las posibilidades del niño y teniendo en cuenta que, firialmente, éste es quién selecciona y elabora en función de sus capacidades. Esto corrobora lo afirmado anteriormente "el scaffolding no sustituye la acción individual pero puede contribuir a su organización y proyección".

2. La siguiente pregunta se centraba sobre la incidencia que tiene la actividad del niño en la organización de la interacción y cómo la organización de la interacción incide en la actividad del primero.

Frente a esta cuestión, los resultados muestran que la significación es necesaria para que el niño inicie un curso de actividad guiado y organizado. A la vez, 
la actividad del niño, es necesaria para que el adulto pueda obtener información relevante para evaluar en qué nivel de significación se encuentra el sujeto, y de este modo, situarse en él ajustándose al proceso interactivo. Además, necesita atender a la actividad del niño como respuesta a su información para conocer su eficacia. Esta es una de las maneras como la interacción incidirá sobre la acción del sujeto, modulándola. Una ruptura en este proceso, bloquearía el feed-back en la interacción.

3. Respecto a las "Condiciones y tipos de interacción", en esta investigación se ha estudiado un solo adulto, por lo tanto, no podemos hablar de diferentes "tipos de adulto", pero sí que se detectan ajustes diferenciales de éste respecto a los niños, lo cual determina distintas formas de intervención.

Estas distintas formas de intervención, por lo tanto, no dependen exclusivamente del adulto, sino de la interacción misma puesto que dicho adulto, en ocasiones, es incapaz de situarse en la perspectiva del niño en cuanto a su conocimiento del objeto y su significado.

4. La última cuestión versaba sobre los efectos de la interacción en el desarrollo cognitivo del sujeto.

Los resultados muestran que no hay efectos positivos de la interacción del adulto sobre el desarrollo del niño si no existe un doble ajuste de aquél tanto al curso de la actividad como al nivel de significación del niño.

Es necesario que el adulto se sitúe en la zona "próxima de significación", si desea favorecer las ganancias de significación en el niño.

Hablamos de una "zona próxima de significación", incluyéndola como elemento trascendental para que el "scaffolding" sea efectivo. El adulto no sólo debe atender al paso de los conocimientos inter-intrapersonales, sino que además debe atender al significado (propio -intra- y del niño -inter-) en ellos implicado.

Queda demostrado que el sujeto participa activamente en la dinámica interactiva, es decir que no todo es interpsicológico, sino que existe una actividad intrapsicológica que determina y condiciona el espacio interpsicológico.

Hay, no obstante, numerosos puntos por resolver (teórico-metodológicos), de entre los cuales citamos:

- la necesidad de profundizar en el tipo de análisis microgenético secuencial de la actividad para conocer mejor su génesis y desarrollo;

- precisar los intercambios indirectos y los mecanismos de control que los niños pueden utilizar en ellos;

- precisar los grados de participación e intercambio de los sujetos como función de la dinámica que se establece.

Estos puntos conforman el cuerpo de los trabajos que actualmente abordamos.

\section{Bibliografía}

ARNAY,j. ET ALT. (1994). Interacción y construcción del conocimiento en bebés: buscando el origen de las teorías implícitas infantiles. Ponencia presentada en el I Simposium sobre Interacción social y Comunicativa en el desarrollo humano. Gerona, 15-17 Diciembre de 1994. 
BEAUDICHON, J., VERBA, M., WINNYKAMEN, F. (1988).Interaction sociale et acquisition de connaissances chez l'enfant: une approche pluridimensionnelle. Revue International de Psychologie Sociale. 1, 131-141.

CAMBRODÍ, A. y SASTRE, S. (1993). Escales d'Observació Sistemàtica: 0-3 anys. Barcelona: P.P.U.

CAREY, S. y GELMAN, R. (Eds.) (1991). The Epigenesis of Mind: Essays on Biology and Cognition. Hillsdale, NJ: LEA.

CARUGATI, F. y GILLY, M. (1993). The Multiple Sides of the same tool: Cognitive Development as a Matter of Social Constructions and Meanings. European Journal of Psychology of Education, III, 4, 343-354.

ELBERS, E. (1991). The development of competence and its social context. Educational Psychology Review, 3, 73-94.

ELBERS, E. (1992). Internalization and adult-child interaction. Learning and Instruction, 2, 101-118.

FRAYSSE, J.C. (1993). Processus cognitif et dynamique interactive. Ponencia presentada en el Workshop "Analyse des dynamiques interactives: pourquoi? comment?". Paris: CNRS.

GILLY,M., BLAYE, A. Y ROUX, J.P. (1988). Elaboración de construcciones cognitivas individuales en situaciones sociocognitivas de resolución de problemas. En G.Mugny y J.A.Pérez (Eds.), Psicología Social del desarrollo cognitivo, 139-164. Barcelona: Anthropos.

GILLY, M. y ROUX, J.P. (1993). Social Routines, Pragmatic Schemas, and Schemas in How Children Distribute Objects Between the Ages of Three and Six. European Journal of Psychology of Education, Vol.XIII, 4, 403-421.

GRÉCO, P. (1991). Structures et significations. Approches du développement cognitif. Paris: Editions de l'Ecole des Hautes Etudes en Sciencies Sociales.

HOUDÉ, O. (1994). Pensée logico-mathématique. Paris: PUF.

INHELDER, B. Y CAPRONA, D. (1992). Un parcours de recherche. En B.Inhelder, G. Céllerier et alt.,Le cheminenment des découvertes de I'enfant. 51-92. Paris: Delachaux et Niestlé.

INHELDER, B., CÉLLERIER, G. et alt. (1992). Le cheminement des découvertes de l'enfant. 119-138. Paris: Delachaux et Niestlé.

KARMILOFF-SMITH, A. (1992). Auto-organización y cambio cognitivo. Substratum, 1,1, 19-43.

LANGER, J. (1980). The origins of Logic. 6 to 12 monts. New York: Academic Press. LANGER, J. (1986). The origins of Logic. One to two years. New York: Academic Press. LANGER, J.(1990). Early cognitive development: Basic functions. En C.A. Hauert (Ed.), Developmental psychology: Cognitive, perceptuo-motor and neuro-psychological perspectives. 19-42. Amsterdam: North Holland.

LANGER, J. (1995). The development of Logic. En V.S. Ramachandran (Ed.). Encyclopedia of Human Behavior. San Diego: Academic Press.

LÉCUYER, R. (1989). Bébés astronomes, bébés psychologues. L'intelligence de la première année. Bruxelles: Pierre Mardaga.

LEHALLE, H. (1990). Les necessités d'un structuralisme ouvert. Archives de psychologie, $58,151-164$. 
MAGNUSSON, M. (1993). Thème behavior resarch software. Reykjavik:Unpublished Manuscript University of Iceland.

MANDLER, J. (1992). How to build a baby : II. Conceptual primitives. Psychological Review, 99, (4), 587-604.

MOUNOUD, P. (1992). L'emergence de conduites novelles: Rapports Dialectiques entre systemes de Connaissances. En G.J.P.Savelsbergh (Ed.). The development of coordination in infancy. Amsterdam: North Holland.

MUGNY, G. y PEREZ, J.A. (Eds.) (1988). Psicología Social del desarrollo cognitivo, 139164. Barcelona: Anthropos.

PASTOR, E. y SASTRE, S. (1994). Desarrollo de la Inteligencia. En V. Bermejo (Ed.) Desarrollo Cognitivo. Madrid: Síntesis,

PASTOR, E. y SASTRE, S. (1995). Patrones de interacción adulto-niño en la construcción del significado: aplicación del programa "Thème". En: Ma ${ }^{a}$ T. Anguera (dir.) Metodología Observacional. Vol.III. Barcelona: P.P.U.

PERRET-CLERMONT, A.N. (1993). What is that Develpops?. Cognition and Instruction, 11 (3\&4), 197-205.

PIAGET, J. y GARCÍA, R. (1987). Vers une logique des significations. Ginebra: Murion Editeur. (Trad. cast. Hacia una lógica de significaciones. Barcelona: Gedisa, 1991).

RIVIÈRE, A-. (1990). Origen y desarrollo de la función simbólica en el niño. En J. Palacios, C. Coll y A. Marchesi (Comps.). Desarrollo psicológico y educación (Vol. 1), 113-132. Madrid: Alianza Psicología.

ROGOFF, B. (1990). Apprenticeship in thinking: Cognitive development in social context. NY: Oxford University Press.

SAADA-ROBERT, M. (1992). La construction microgénétique d'un schème elementaire. En B.Inhelder, G. Céllerier et alt. Le cheminenment des découvertes de l'enfant. 119-138. Paris: Delachaux et Niestlé.

SASTRE, S. (1991). Estudio comparativo sobre la influencia del adulto en la actividad cognitiva del niño trisómico-niño normal entre $0 ; 11$ a.1;6a. Tesis Doctoral no publicada. Universidad de Barcelona

SASTRE, S. y PASTOR, E. (1992). Vers una interpretació constructiu-contextualista de la deficiència: I'adult com a mediador. Revista de Psicologia Universitas Tarraconensis. XIII (1), 125-134.

SASTRE, S. y PASTOR, E. (1993). Une proposition méthodologique pour l'étude de l'organisation logique de l'activité individuelle et interactive. Ponencia presentada en el Workshop "Analyse des dynamiques interactives: pourquoi? comment?". Paris, CNRS.

SASTRE, S. y PASTOR, E. (1994). Action, Signification process and Scaffolding organization. Poster presented to the XIIIth Biennal Meetings of ISSBD. Amsterdam.

SASTRE, S. y PASTOR, E. (en prensa). Desarrollo Cognitivo y organización lógica de la Acción: propuesta de análisis cualitativo. En $M^{\mathrm{a}} \mathrm{T}$. Anguera (dir.). Observación en la escuela. Barcelona. Paidós.

SPELKE, E. (1991). Physical Knowledge in Infancy: Reflections on Piaget's Theory. En S.Carey y R.Gelman (Eds.). The Epigenesis of Mind: Essays on Biology and Cognition. 133-170. Hillsdale, NJ: LEA.

SPELKE, E. (1994). Initial knowledge: six suggestions. Cognition, 50, 431-445. 
STERNBERG, R.J. (1993). Metaphors of Mind. Conceptions of the Nature of Intelligence. Cambridge: Cambridge University Press.

TUDGE, J. \& WINTERHOFF, P. (1993). Vygotsky, Piaget and Bandura: Perspectives on the Relations between the Social World and Cognitive Development. Human Development, 794.

VERBA, M. (1993a). Discussion. International Journal of Psychology, 28 (5), 671-675.

VERBA, M. (1993b). L'analyse des dynamiques interactives. Ponencia presentada en el Workshop "Analyse es dynamiques interactives: pourquoi? comment?". Paris, CNRS.

VERBA, M. (1994). The beginnings of collaboration in Peer interaction. Human Development, 37, 125-139.

WERTSCH,J.V. (Ed.) (1985). Culture, communication and cognition, vygotskian perspectives. New York: Cambridge University Press.

WOOD, D., BRUNER, J. y ROSS, T.G. (1987). The role of tutoring in problem-solving. Journal of Child Psychology and Psychiatry, 17, 89-100.

\section{ANEXO 1. Códigos de Categorías}

ACCIÓN DEL NIÑO (An)

Ano No acción física

An1 Acción intra-objeto

An2 Acción inter-objetos

An5 Acción verbal

SIGNIFICACION DEL NIÑO (Sn) ( Piaget \& Garcia, 1987)

SnO No significación

Sn1 Atributiva local

Sn2 Atributiva sistémica

Sn3 Atributiva estructural

Sn4 Integrativa local

Sn5 Integrativa sistémica

Sn6 Integrativa estructural

Sn7 Significación de significaciones

MODELO ADULTO(M)

MO Ausencia de modelo

M1 Modelo físico ajustado a la acción del niño.

M2 Modelo físico no ajustado

M3 Modelo verbal ajustado

M4 Modelo verbal no ajustado

M5 Modelo físico y verbal ajustado

M6 Modelo físco y verbal no ajustado

M7 Confirmación verbal del resultado del niño.

SIGNIFICACION MEDIADA DEL ADULTO (Sa)

SaO No significación

Sa1 Atributiva local

Sa2 Atributiva sistémica 
Sa3 Atributiva estructural

Sa4 Integrativa local

Sa5 Integrativa sistémica

Sn6 Integrativa estructural

Sa7 Significación de significaciones

ACCION RESULTANTE (Ra)

Ra0 Ausencia de acción resultante

Ra1 No ganancia en la acción resultante.

Ra2 De la exploración indiferenciada a la diferenciada.

Ra3 De la exploración diferenciada a la experimentación

Ra4 De la exploración sin generalización a la generalización.

Ra5 Nueva composición

SIGNIFICACION RESULTANTE (Rs)

Rs0 Ausencia de significación resultante

Rs1 Ausencia de ganancia en la significación resultante.

Rs2 Del no conocimiento a la significación atributiva estructural

Rs3 De la significación atributiva estructural a la significación integrativa.

Rs4 Ganancia en la significación integrativa

Rs5 De la significación integrativa a la significación de significaciones.

\section{ANEXO 2. Definición de algunas categorías de "Significación"}

SIGNIFICACION (S) - NIÑO (Sn) / ADULTO (Sa)

SIGNIFICACIÓN

(Ver definición en el marco teórico del estudio empírico)

NO SIGNIFICACIÓN (SnO)

(+) Se entiende que no hay significación cuando el sujeto ante un objeto, aunque dé muestras de interés ante el mismo, no aplica un curso de acción organizado sobre él.

Ejemplo: niño ante objetos, mira objetos, coge cilindro, mira objetos, cae cilindro...

(?) Es dudoso cuando el niño aplica un esquema conocido al objeto por conocer. En este caso, el significado estaría en el esquema y no en el objeto.

Siempre que la aplicación del esquema sea puntual y no vaya seguida de otros esquemas con monitorización visual, no puede justificarse la existencia de significado.

Ejemplo: niño coge objeto, sacude objeto, deja objeto, mira otros objetos...

Significación atributiva

(+) Es aquella en la que existe un predominio de los esquemas de acción básicos, de los que dispone el sujeto, sobre las propiedades del objeto.

Consiste en aplicar esquemas conocidos a objetos nuevos (desconocidos). La trayectoria cognitiva va desde el esquema conocido al objeto por conocer.

Ejemplo: el niño coge pieza-base, mira, sacude, introduce dedo en hueco central, mira, sacude,... 
(-) Ver definición significación integrativa como criterio diferencial opuesto a significación atributiva.

(?) En caso de duda cuando el niño realiza una modularización sucesiva del esquema de acción. En este caso, no hay significación ya que ello supone, no una exploración del objeto, sino que la actividad está centrada en la optimización de la acción en sí misma.

Ejemplo: ante un instrumento con que se puede coger un objeto (vara), el niño aplica distintas tentativas de presión con observación sucesiva de la mano.

\section{Significación Integrativa}

Es aquella en la que existe un predominio de las propiedades de un objeto sobre los esquemas de acción básicos de los que dispone un sujeto.

Las propiedades del objeto, en sí mismo, condicionan y determinan diferencialmente los esquemas a aplicar sobre sí mismo.

La trayectoria cognitiva en este caso va, desde el objeto conocido hacia el/los esquema/esquemas a aplicar (diferencialmente).

Ejemplo: niño coge rueda, mira rueda, gira, rueda, observa.

(-) Ver definición de significación atributiva.

(?) Esta categoría reclama un conocimiento del objeto, por lo tanto una de las dudas consiste en saber si el objeto es conocido.

Los indicadores que permiten afirmar la existencia de dicho conocimiento son:

* no hay tanteos en la acción del sujeto.

* Ios esquemas aplicados se adecuan a las propiedades del objeto. Pueden modular dichos esquemas, pero siempre son adecuados desde su primera aplicación.

Significación atributiva Local (sn1 - Sna1)

Se trata de una categoría con el grado más elemental de significación. Supone el establecimiento de la significación a partir de los resultados constatados y en contextos limitados o particulares, teniendo en consideración un número limitado de datos sobre los objetos.

Por lo tanto, surge a través de la aplicación de los esquemas básicos de acción y tiene como resultado la obtención de la información parcial del objeto, SIN illegar al establecimiento de los POSIBLES.

Es, por tanto, una actividad básicamente de PRIMERA EXPLORACIÓN del objeto a conocer.

Ejemplo: niño coge pieza-base, mira pieza-base, gira pieza-base, mira, pone dedo en hueco central, mira, saca dedo de hueco central, mira, pone dedo en hueco lateral.

(-) Tanto la falta de significación como la existencia de un nivel de significación superior al descrito supone la negación de éste. 
REVISIÓN Y DEBATE 
